\title{
Hippocampal Formation Size in Normal Human Aging: A Correlate of Delayed Secondary Memory Performance
}

James Golomb, ${ }^{1,5}$ Alan Kluger, ${ }^{2}$ Mony J. de Leon, ${ }^{2}$ Steven H. Ferris, ${ }^{2}$ Antonio Convit, ${ }^{2}$ Mary S. Mittelman, ${ }^{2}$ Jacob Cohen, ${ }^{3}$ Henry Rusinek, ${ }^{4}$ Susan De Santi, ${ }^{2}$ and Ajax E. George ${ }^{4}$

${ }^{1}$ Departments of Neurology, ${ }^{2}$ Psychiatry, ${ }^{3}$ Psychology, and ${ }^{4}$ Radiology

Aging and Dementia Research Center

New York University Medical Center

New York, New York 10016

\section{Abstract}

\begin{abstract}
Although mild progressive memory impairment is commonly associated with normal human aging, it is unclear whether this phenomenon can be explained by specific structural brain changes. In a research sample of 54 medically healthy and cognitively normal elderly persons (ages 55-87, $\bar{x}=69.0 \pm 7.9$ ), magnetic resonance imaging (MRI) was used to derive head-size-adjusted measurements of the hippocampal formation (HF) (dentate gyrus, hippocampus proper, alveus, fimbria, subiculum), the superior temporal gyrus (STG), and the subarachnoid cerebrospinal fluid (CSF) (to estimate generalized cerebral atrophy). Subjects were administered tests of primary memory (digit span) and tests of secondary memory with immediate and delayed recall components (paragraph, paired associate, list recall; facial recognition). Separate composite scores for the immediate and delayed components were created by combining, with equal weighting, the subtests of each category. The WAIS vocabulary subtest was used as a control measure for language and intelligence. A highly significant correlation $(P<0.001)$, independent of age, gender, and generalized cerebral atrophy was found between HF size and delayed memory
\end{abstract}

\footnotetext{
${ }^{5}$ Corresponding author.
}

performance. No significant correlations were found between HF size and primary or immediate memory performance. STG size was not significantly correlated with any of the composite memory variables. These results suggest that HF atrophy may play an important independent role in contributing to the memory loss experienced by many aging adults.

\section{Introduction}

A decline in the ability to remember recently presented information following a time delay (delayed secondary memory) is recognized to occur in normal human aging (Fozard 1985; Poon 1985). Although the precise neuroanatomic basis for this phenomenon is unknown, progressive dysfunction of the hippocampal formation (HF) has been proposed as a possible mechanism (Moscovitch and Winocur 1992). The HF is a central component of the medial temporal lobe memory system, and its structural integrity is necessary for effective delayed declarative memory processing (Squire and Zola-Morgan 1991). Neuroradiographic evidence for HF tissue loss has been described in human disease states associated with severe memory impairment (de Leon et al. 1989; Press et al. 1989; Jack et al. 1992). Similar changes have been observed in patients with mild Alzheimer's disease as well as in elderly individuals exhibiting cognitive impairment suggestive of questionable dementia (Convit et al. 1993; de Leon et al. 1993; Killiany et al. 1993). Nevertheless, it is uncertain to what ex-

LEARNING \& MEMORY 1:45-54 당 1994 by Cold Spring Harbor Laboratory Press ISSN1072-0502/94 \$5.00

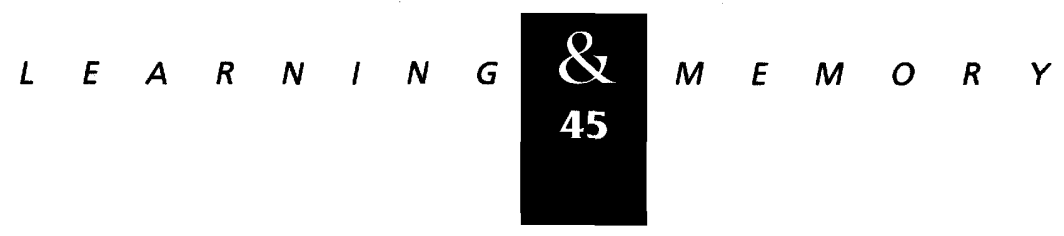


tent atrophy of this structure can account for the milder decline in memory performance experienced by many neurologically healthy older persons.

Using subjective techniques of X-ray computed tomography (CT) and magnetic resonance imaging (MRI) interpretation, we recently showed that medial temporal lobe atrophy in the region of the hippocampus is common in cognitively normal elderly individuals and that the presence of such atrophy is associated with diminished performance on tests of delayed memory (Golomb et al. 1993). Nevertheless, because diffuse gyral shrinkage also occurs with aging (Kemper 1984), these findings do not support a "unique" association between HF atrophy and the observed memory change. Techniques of MRI morphometry make it possible to obtain quantitative in vivo size measurements of the $\mathrm{HF}$ and other brain structures (Jack et al. 1990; Convit et al. 1993). Using these techniques, we now provide evidence that HF size is a significant independent predictor of delayed memory function in normal human aging.

In studies of age-related structural brain change, the demonstration of relationships between specific anatomic regions and behavior requires an experimental design that controls for the effects of diffuse cerebral atrophy. Radiologic studies based on sulcal cerebrospinal fluid (CSF) measurements suggest that the Sylvian fissure and frontoparietal vertex sulci are sensitive regions for detecting age-associated gyral shrinkage (Pfefferbaum et al. 1990). Histopathologic studies of normal aging support this observation by demonstrating a significant loss (or shrinkage) of large neurons within the precentral, postcentral, and superior temporal gyri (Kemper 1984). Comparable levels of age-associated neuronal change have also been described for the lateral temporal lobe, a site of early neocortical pathology in Alzheimer's disease (Kemper 1984). Because these regions have no primary memory function, but nevertheless show considerable atrophy, they are ideally suited to test the specificity of a possible relationship between HF size and memory performance.

In this study our objective was to investigate whether in normal human aging a significant correlation exists between HF size and delayed memory performance that is independent of the ageassociated atrophy affecting brain regions without direct memory processing significance. To this end, we used statistical methods of multiple linear regression to test two related hypotheses. (1) In a sample of cognitively normal elderly persons, HF size is a significant predictor of delayed memory performance even after partialling out the combined effects of age, gender, and diffuse cerebral atrophy. (2) The size of a lateral temporal lobe structure without direct memory processing importance is not a significant predictor of memory performance. The superior temporal gyrus (STG) was chosen for this latter purpose because of four considerations. First, its neuropsychologic role is believed to be relatively independent of memory function (Kolb and Whishaw 1990). Second, histopathologic studies of normal aging suggest that it may be among the most severely affected gyri in terms of neuronal loss (Brody 1955; Terry et al. 1987). Third, it is easily identified, and fourth, its long axis runs parallel with that of the HF, permitting the two structures to be adequately measured on the same set of cross-sectional images.

\section{Materials and Methods}

Fifty-four medically healthy and cognitively normal individuals 55 years of age and older ( 23 males, 31 females, age range 55-87 years, mean $=69.0$, S.D. $=7.9$ ) were consecutively selected from a larger pool of community-dwelling research volunteers participating in a study of normal aging. Forty-four of these subjects participated in our previous study using subjective ratings of medial temporal lobe atrophy (Golomb et al. 1993). Selection was based on the following inclusion and exclusion criteria.

\section{INCLUSION CRITERIA}

Inclusion was contingent on a rating of 1 or 2 on the Global Deterioration Scale (GDS) (Reisberg et al. 1982), as well as a score of 28 or higher on the Mini Mental Status Examination (MMSE) (Folstein et al. 1975). The MMSE is a widely accepted brief screening instrument for serious cognitive impairment with a maximum score of 30 . A score of 23 or below is considered suggestive of dementia (Cockrell and Folstein 1988). The GDS is a seven-point scale for staging cognitive, psychiatric, and functional impairment in patients with Alzheimer's disease. In clinical studies of dementia, GDS ratings of 1 and 2 are used to define samples of cognitively normal control subjects. Such subjects are unlikely to undergo significant cognitive deterioriation within a 3- to 4-year interval (Flicker et al. 1993). In this study the inclusion only of cog-

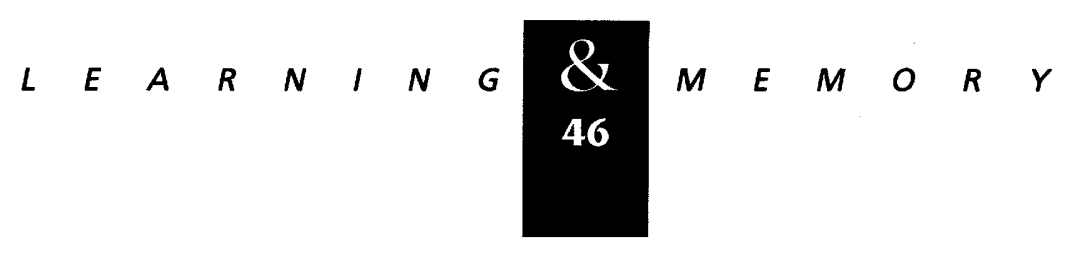


nitively normal cases was further ensured by requiring MMSE scores of 28 and above.

\section{EXCLUSION CRITERIA}

All subjects received comprehensive medical, neurologic, psychiatric, neuroradiologic (brain MRI), and laboratory examinations (blood and urine). Exclusions were made if any of these examinations suggested the presence of a disease state that could affect brain functioning. Specifically, subjects were excluded if there was any clinical or radiographic evidence for structural, metabolic, or epileptogenic central nervous system abnormalities, including cerebral infarction (cortical or lacunar), severe leukoaraiosis (Hachinski et al. 1987), or if the modified Hachinski Ischemia Scale rating (Rosen et al. 1980) was $>3$. Also excluded were persons with more than borderline hypertension $(>160 / 90 \mathrm{~mm} \mathrm{Hg})$ or significant cardiovascular, rheumatologic, endocrinologic, hematologic, neoplastic, pulmonary, or psychiatric disorders. Subjects were also excluded if they received a Hamilton Depression Scale score (Hamilton 1967) of 16 or greater, if there was a history of excessive alcohol intake, or if they were taking any medication affecting cognition. The demographic characteristics of the study are described in Table 1.

\section{MRI EVALUATIONS}

For each subject, MRI was used to generate a set of coronally oriented 4-mm-thick, T1-weighted images through the temporal lobe. All scans were acquired on a 1.5 tesla Philips Gyroscan (Shelton, CT). There was a $10 \%$ gap between slices, and the scan specifications were as follows: $23-\mathrm{cm}$ FOV, $516 / 21 / 2$ (TR/TE/excitations), acquisition matrix $204 \times 256$. A set of 18 images angled 90 degrees to the long axis of the hippocampus as determined on a T1-weighted sagittal scout was obtained through the temporal lobes. These slices were also perpendicular to the long axis of the STG and included the CSF anatomy of the Sylvian fissure.

Slices depicting the body of the hippocampus from immediately posterior to the hippocampal head to the level of the posterior pulvinar were used to measure the size of the HF, the STG, and the subarachnoid cerebrospinal fluid (SCSF) compartment. The SCSF served as an index of generalized temporal, perisylvian, and frontoparietal gyral atrophy. On each of these slices, the intracranial supratentorial (ST) compartment was also measured as an estimate of head size. These measurements were performed on the digitized images using a Sun work station (Sun Microsystems Inc., Mountain View, CA) and software that permits the tracing of anatomic regions, the selection of pixels based on gray-scale intensity levels, and the determination of a region's size based on the number of pixels so identified (see Fig. 1).

In this study the right and left HF region was defined by inclusion of the following anatomic structures: alveus, fimbria, dentate gyrus, hippocampus proper, and the section of subiculum occupying the most superior portion of the parahippocampal gyrus. T1-weighted MRIs permit gray and white matter structures to be differentiated, but histologic features separating the cortical subdivisions of the parahippocampal gyrus cannot be discerned. These considerations combined with limitations in MRI spatial resolution prevented us from reliably identifying such regions as the entorhinal cortex and made it necessary to operationally define the medial extent of the HF region as described.

With reference to a neuroanatomic atlas, the right and left STG was also outlined. This region was drawn to include a wedge-shaped area defined by the center of the temporal horn and the superior and inferior sulcal borders of the STG (Convit et al. 1993; see Fig. 1). The intracranial (ST) compartment was defined by tracing the inner table of the calvarium and the tentorium cerebelli. On

Table 1: Demographic and clinical characteristics $(n=54)$

\begin{tabular}{ll}
\hline Male (\%) & 42.6 \\
Subjective memory complaints (GDS 2) (\%) & 90.1 \\
Mean ( \pm S.D.) age (years) & $69.0(7.9)$ \\
Mean ( \pm S.D.) years of education & $15.0(2.7)$ \\
Mean ( \pm S.D.) MMSE score & $29.4(0.6)$ \\
Mean ( \pm S.D.) WAIS vocabulary score & $66.0(11.4)$
\end{tabular}

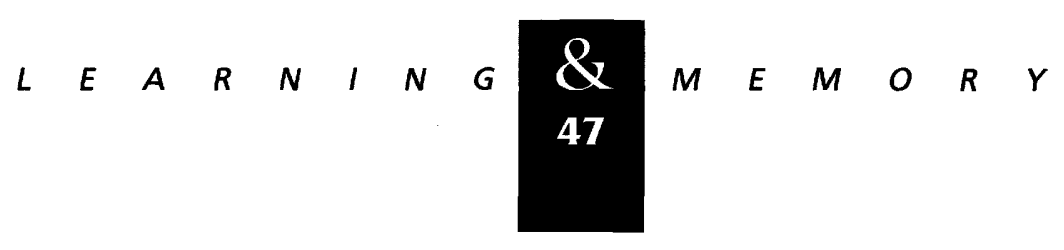


Golomb et al.
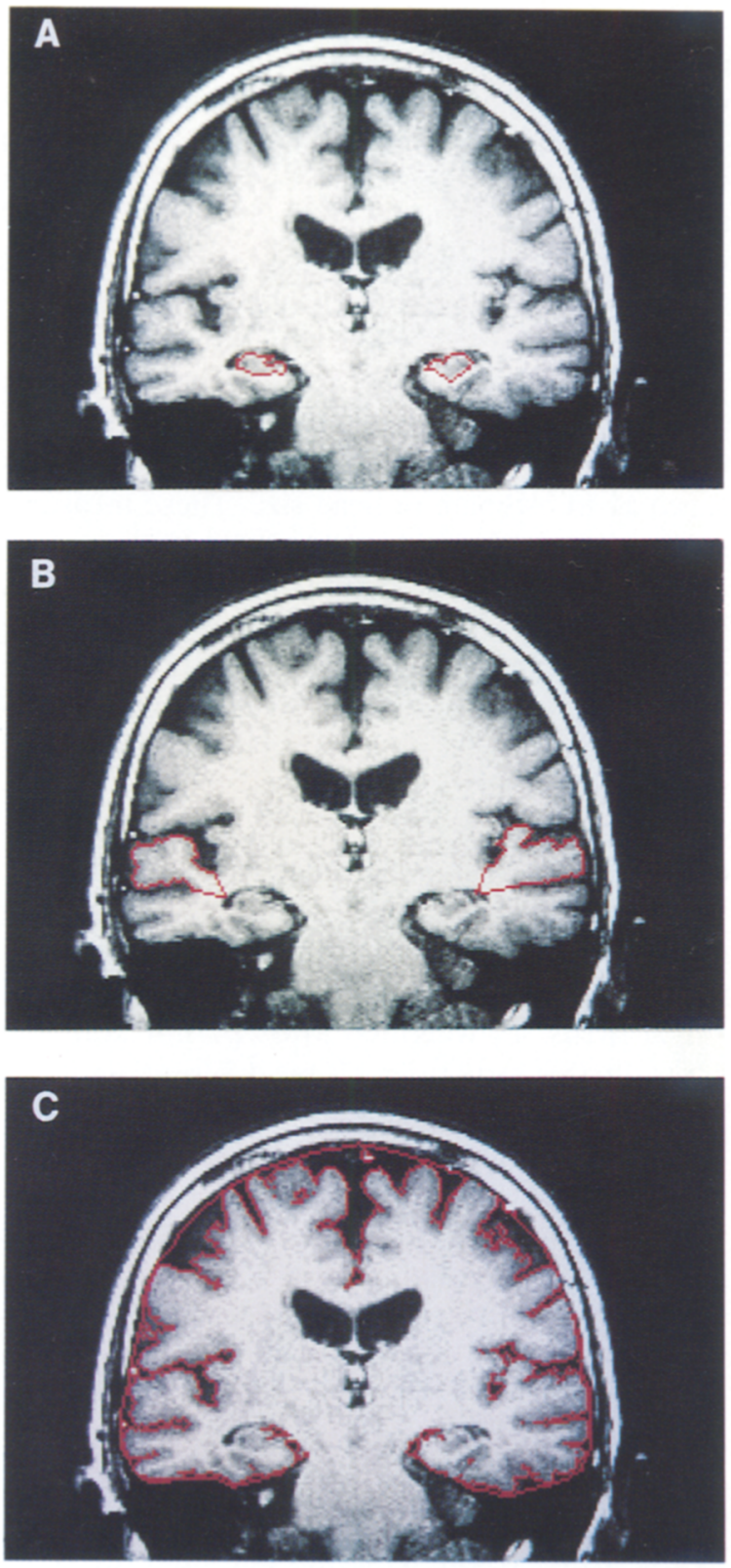

Figure 1: A coronal T1-weighted magnetic resonance image illustrating the three brain regions used as independent variables in the multiple regression analysis. (A) HF; (B) STG; (C) SCSF. The size of each region is determined by the number of pixels falling within the red boundaries (see text for methodology). For this representative case, the head size-residualized scores corresponding to these regions fall within 0.5 S.D. of the mean for the entire sample ( $\mathrm{rHF}=-2.46,0.18$ S.D. below mean; $r S T G=-25.64,0.39$ S.D. below mean; $\mathrm{rSCSF}=-8.44,0.01$ s.D. below mean.

each slice, the pixel intensity value falling midway between the mean of a non-partial-volumed ven- tricular CSF sample and the mean of the HF sample was calculated. This value was used as a threshold for separating CSF pixels from brain parenchyma pixels within the ST. SCSF was then derived by selecting only those CSF pixels that were not part of the ventricular system. This threshold was also used to exclude CSF pixels from the STG regions. Because both the right and left hippocampi contribute to memory performance and because they are correlated in size $(r=0.76, P<0.001)$, a mean of the right and left pixel counts for the HF and STG was calculated and used in the statistical analysis.

All region analysis was performed retrospectively without knowledge of the subject's age or clinical status by a single observer with demonstrated reliability in making these measurements. In an independent reanalysis of $\mathbf{2 4}$ randomly selected cases, this observer reproduced the original HF measurements with a mean deviation (RMS) of 2.65 pixels. This level of error is $<3 \%$ of the mean HF pixel count for the entire sample (103.41). The general validity and reproducibility of MRI morphometry using nearly identical techniques have also been demonstrated by others (Jack et al. 1990).

For each case, mean pixel counts per slice were calculated for the HF, STG, and SCSF. To adjust for intersubject head-size variability, these values were each regressed on the mean ST pixel count to generate head-size-residualized scores (rHF, rSTG and rSCSF) that were then used in subsequent statistical analyses (Sullivan et al. 1993). To achieve the largest possible sample for this head-size correction analysis, 22 additional subjects were included (total $n=76$ ). These subjects met the same selection criteria described previously but were not included in the main analyses because they underwent MRI scanning prior to the introduction of the specific memory tests that formed the basis of this study. Differences in HF size for three representative cases are illustrated in Figure 2.

\section{PSYCHOMETRIC EVALUATIONS}

Subjects were administered a battery of six memory tests comprised of (1) paragraph recall from the Guild Memory Test (Gilbert et al. 1968), (2) word-list recall from the Shopping-List Task (Ferris et al. 1988), (3) facial recognition from the Facial Recognition Task (Ferris et al. 1980), and (4) paired associate recall from the Guild Memory

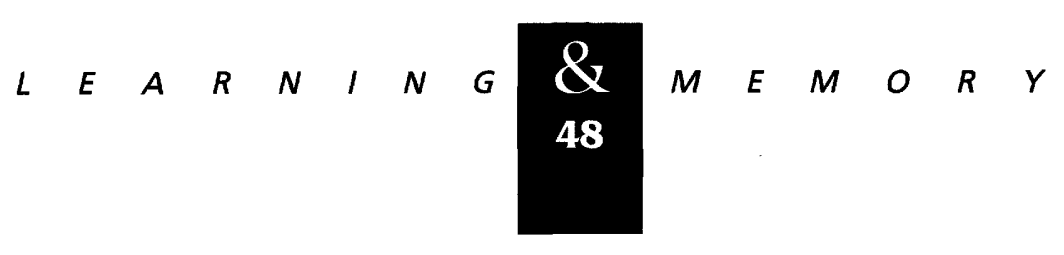




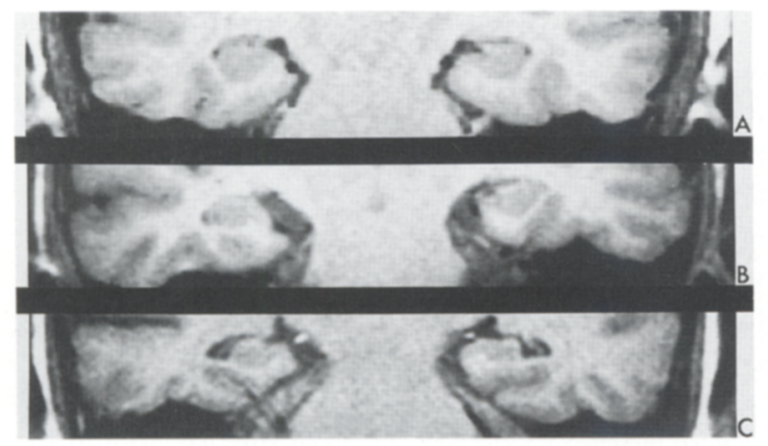

Figure 2: Coronal T1-weighted magnetic resonance images of three subjects highlighting the inferior temporal lobe anatomy to illustrate gradations of HF size. (A) $\mathrm{rHF}=15.16,1.41$ S.D. above mean; $(B) \mathrm{rHF}=8.79$, 0.83 s.D. above mean; (C) rHF $=-10.26,0.88$ s.D. below mean.

Test, the First-Last Names Task [Ferris et al. 1986 (as modified from French et al. 1974)], and the Name-Face Task (Ferris et al. 1986). For each of these tests, a score measuring the amount of information remembered immediately after presentation of the test material (initial recall) and a score measuring recollection following a time delay (delayed recall) were obtained. Each test had a standard delay interval; across tests, the delay range was 5-30 min.

Separate composite scores for initial recall (IR) and delayed recall (DR) were created by combining, with equal weighting, the subtests of each category as follows: Raw scores for both the immediate and delayed components of each of the six memory tests were transformed to standardized scores ( $\mathrm{z}$ scores). IR and DR were then computed as the unweighted mean of the immediate and delayed standardized scores, respectively. Creating composite indices by averaging different measures of the same category of memory function permits the number of psychometric variables in the statistical analysis to be reduced and allows a more reliable representation of performance within each category to be achieved. Some of the memory tests used to form IR and DR included additional learning trials following initial recall and prior to the delay interval. In the interest of developing composite measures that optimize the distinction between immediate and delayed memory, data from these learning trials were omitted from the current analyses.

In addition, all subjects received a test of forward digit span (DIG) as a pure measure of shortterm (primary) memory (Wechsler 1958). Be- cause of the predominately verbal nature of the memory tests, the vocabulary subtest (VOC) of the Wechsler Adult Intelligence Scale (WAIS) was provided as a control measure of language and intelligence (Wechsler 1958). Scores on this test are highly correlated with the full-scale IQ and remain relatively stable in normal aging (Matarazzo 1972). Raw scores from both DIG and VOC were utilized in the statistical analyses.

In humans and nonhuman primates with hippocampal lesions, short-term memory is preserved but recall performance progressively deteriorates in proportion to the time delay following an initial presentation of information (Squire and Zola-Morgan 1991). This observation suggests that DR (and not DIG or IR) should correlate with hippocampal dysfunction in our sample. The DR score, however, is partly dependent on initial recall and short-term memory influences, and as such, it cannot be considered a pure measure of delayed memory function. To remove these influences from the DR score, DR was regressed on IR and DIG; the residual, rDR, was used as a dependent measure of delayed memory performance. [Although a subject's performance on tests of IR reflect information retrieval from both secondary and primary memory stores, the limited number of test items and the short time period required to present the material $(20-50 \mathrm{sec})$ minimize the role of secondary memory processes. It is therefore unlikely that IR would be significantly influenced by mild HF dysfunction].

The tests comprising the composite measures used in this analysis are typical of procedures commonly used in the clinical assessment of human memory performance and have proven useful in detecting memory changes associated with normal aging and early Alzheimer's disease. These tests are routinely administered at our center and are summarized as follows: (1) With the paragraph recall subtest from the Guild Memory Test, the subject is read a short paragraph and then asked to recall as many components as possible. The paragraph is then reread to the subject and DR is assessed after $5 \mathrm{~min}$. Scores on two different paragraphs are averaged to produce separate estimates of initial and delayed recall. (2) With the Shopping-List Task, the subject is sequentially presented with 10 grocery items to read aloud and remember. After each of up to five trials (presentation and free recall), the subject is reminded of the items not recalled. The learning trials are terminated after two successive omission-free trials. Delayed recall is as-

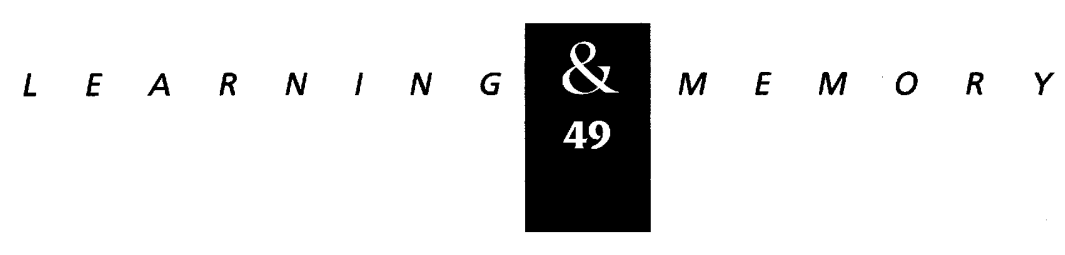


sessed $30 \mathrm{~min}$ after the last trial. (3) With the Facial Recognition Task, the subject is sequentially presented with 10 photographs of unfamiliar faces. The photographs are then represented along with 10 new photographs in random order. Recognition for the target photographs is tested over three trials, followed by a delayed trial $30 \mathrm{~min}$ later. (4) With the Paired Associate Recall of the Guild Memory Test, the subject is sequentially read 10 word pairs that vary in associative value. The first word of each pair is then given and the second word must be recalled. Subjects are reinforced for a correct response and are cued with the appropriate second word for incorrect recall. A delayed recall trial is administered after $5 \mathrm{~min}$. (5) With the Paired Associate Recall of the First-Last Names Task, a list of four first-last name pairs is sequentially presented. The subject is then tested for recall of the first name in response to the presentation of the last name. Up to three trials are administered, with a learning criterion of two consecutive error-free trials. A delayed recall trial is administered after $30 \mathrm{~min}$. (6) The Paired Associate Recall of the Name-Face Task is identical to the First-Last Names Task described above, except that the paired associates consist of a first name and a photograph of a unfamiliar face. Test trials involve presentation of the faces alone, and the subject must recall the correct name.

\section{Results}

In a series of linear regression analyses, we first examined the relationship of age to each of the head-size-residualized brain regions. Although the age effect for rSTG was considerably weaker than that seen for the other brain regions, these analyses revealed significant negative correlations for both parenchymal structures and a significant positive correlation for $\mathrm{rSCSF} \quad\left(r_{\mathrm{rSTG}}=-0.27\right.$, $P<0.05 ; \quad r_{\mathrm{rHF}}=-0.46, P<0.0001 ; r_{\mathrm{rSCSF}}=0.55$, $P<0.0001)$. These results are consistent with the widely recognized phenomenon of brain volume loss with subarachnoid space expansion that accompanies normal aging.

To examine the specific effect of rHF on memory performance while controlling for the influence of age, sex, language/intelligence, and generalized atrophy, a hierarchical multiple regression statistical model was employed (Cohen and Cohen 1983). With rDR as the dependent variable, age, sex, VOC, and rSCSF were entered at step 1. In accord with our hypothesis, rHF but not rSTG resulted in a significant improvement in $R^{2}$ when added to the equation at step $2\left(\Delta \mathrm{R}^{2}=0.19, P<\right.$ 0.001 ). In parallel analyses with IR and DIG as dependent variables, both rHF and rSTG failed to result in significant $\mathbf{R}^{2}$ changes at step 2 . The distributions of the dependent variables were similar in shape and revealed no evidence for ceiling or floor effects.

The partial correlation coefficients associated with these multiple regression models are provided in Table 2 . In part $A$, only the control variables are used in the equations as predictors. In part $B$, all of the variables are forced into the equations as predictors. In part B, rSCSF and rHF are highly significant as independent brain predictors of DR but only rHF is significant as an independent predictor of rDR. This observation supports our hypothesis that rDR is a more hippocampal-specific memory variable than DR by virtue of its independence from the influences of IR and primary

Table 2: Partial correlation coefficients for multiple regression models

\begin{tabular}{rlrlll}
\hline & Predictors & \multicolumn{1}{c}{$\mathrm{DIG}^{\mathrm{a}}$} & \multicolumn{1}{l}{$\mathrm{IR}^{\mathrm{a}}$} & \multicolumn{1}{l}{$\mathrm{DR}^{\mathrm{a}}$} & \multicolumn{1}{l}{$\mathrm{rDR}^{\mathrm{a}}$} \\
\hline \multirow{4}{*}{ Part A } & VOC & $0.31^{*}$ & $0.45^{* * *}$ & 0.27 & -0.15 \\
& age & -0.24 & $-0.49^{* * *}$ & $-0.50^{* * *}$ & 0.18 \\
& sex & -0.04 & $-0.32^{*}$ & $-0.41^{* *}$ & -0.23 \\
& VOC & $0.31^{*}$ & $0.44^{* *}$ & 0.25 & -0.21 \\
& age & -0.26 & -0.24 & -0.12 & 0.13 \\
Part B & sex & -0.05 & $-0.34^{*}$ & $-0.44^{* *}$ & -0.22 \\
& rSCSF & -0.12 & $-0.38^{* *}$ & $-0.41^{* *}$ & -0.11 \\
& rSTG & 0.06 & 0.03 & 0.10 & 0.10 \\
& rHF & -0.24 & 0.10 & $0.46^{* * *}$ & $0.47^{* * *}$ \\
\hline
\end{tabular}

a* $P<0.05 ;{ }^{* *} P<0.01 ;{ }^{* * *} P<0.001$.

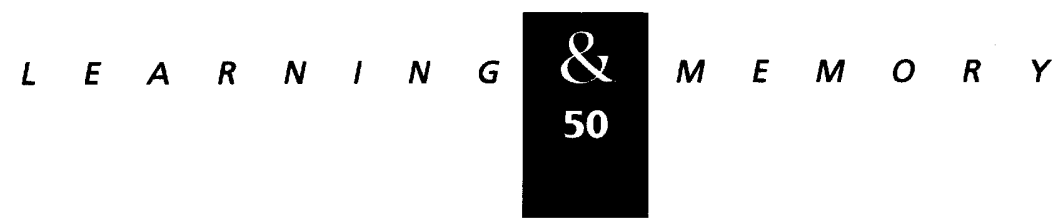


memory (DIG). The linear relationship between rHF and rDR is illustrated in Figure 3. The finding that rSCSF is the only brain measure to significantly predict IR is further evidence that atrophy of nonhippocampal brain regions may explain aspects of memory performance that are less delaydependent. DIG was based on a single rather than a composite measure of primary memory, and this could explain its failure to significantly correlate with rSCSF. Part A demonstrates that in our sample both age and sex (female memory performance $>$ male) are significant independent predictors of DR and IR. Interestingly, addition of the brain region variables to the equation (part B) eliminates the predictive significance of age, with a corresponding reduction of its partial coefficients by $>50 \%$. It appears, therefore, that in this sample the decline in memory performance associated with increasing age can be explained entirely in terms of atrophic brain change. Finally, part A reveals that although age and sex each account for a substantial proportion of the variance in DR, neither of these variables significantly predicts rDR. This suggests that the effects of age and sex on DR may be due largely to their strong independent effects on IR. The absence of a significant association between delayed recall and age after correcting for the influence of initial recall has been observed by others as well (Petersen et al. 1992).

\section{Discussion}

Our results demonstrate that HF size can predict delayed memory performance in a research sample of cognitively normal elderly individuals.
Anatomic specificity for this relationship is suggested by its independence from the effects of diffuse gyral atrophy and by the failure of rSTG to correlate with any of the memory variables, a finding consistent with the lack of a primary memoryprocessing role for this structure. The neuropsychologic specificity for delayed recall is suggested by the absence of a significant correlation between rHF and the two measures of nondelayed memory function, IR and DIG.

In conjunction with our previous study (Golomb et al. 1993), these results now demonstrate that two independently applied and methodologically distinct assessments of HF atrophy produce the same result with respect to delayed memory function in normal aging. Our previous study of 145 identically selected subjects (27\% overlap with the present sample) used subjective ratings of axial CT and MRI scans to show that atrophy of the hippocampal region is associated significantly with delayed secondary memory performance (Golomb et al. 1993). This study confirms this phenomenon with coronal MRI images using a technique that is both quantitative and anatomically specific.

Relatively few of the myriad factors contributing to memory test performance in older individuals can be adequately controlled in an experimental setting. Although we limited our study to a select group of highly screened and well-motivated research subjects, differences in baseline (presenile) memory function, life-span environmental influences, and other psychologic factors unrelated to memory are all sources of rDR variance that could not be assessed independently. Given these potential sources of error, our detection of a statistically significant correlation be-

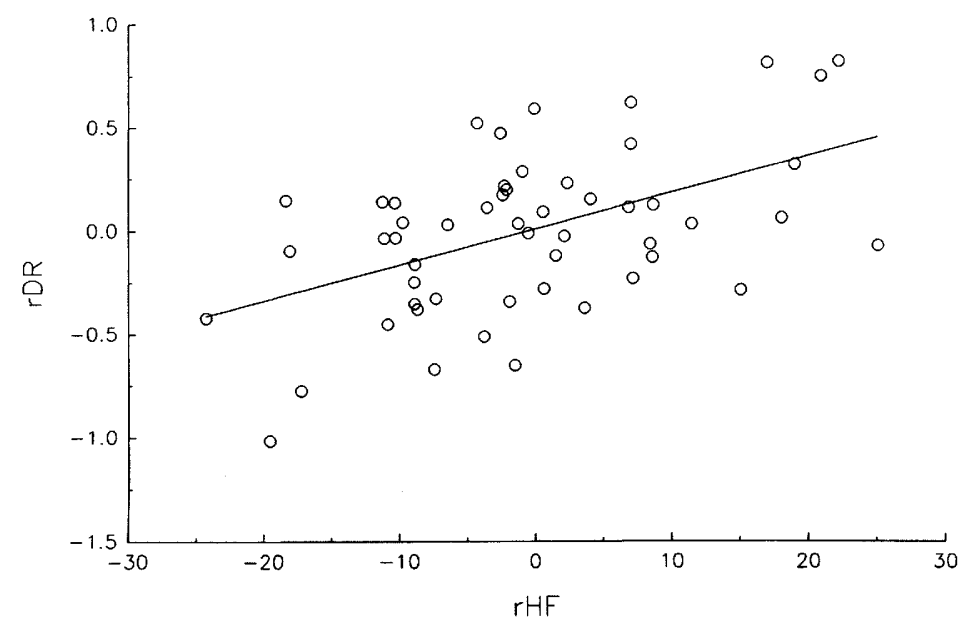

Figure 3: Plot illustrating the linear univariate regression of rDR on rHF. $r=0.49, P<0.001$. This relationship persists even after partialling out variance due to age, sex, VOC, rSCSF, and rSTG (see text; Table 2).

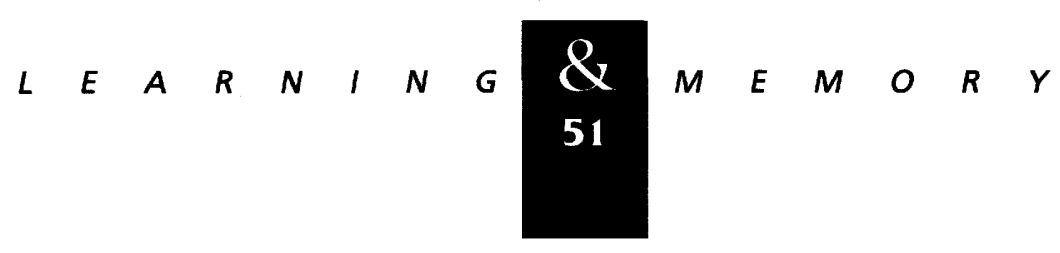


tween HF size and memory performance supports the robustness of this relationship.

Practical constraints limited the scope of our study to a relatively circumscribed set of objectives. In particular, we did not attempt to test double dissociation hypotheses concerning structure/ function relationships of the HF and STG. Furthermore, our study was not designed to examine whether the sizes of brain regions other than the HF and STG correlate with memory performance. Although necessary, an intact HF does not ensure effective delayed memory function. For example, age-associated degenerative processes affecting the frontal lobe may also contribute to memory impairment (Moscovitch and Winocur 1992). Relatedly, it must be emphasized that atrophy of other components of the medial temporal lobe memory system may be even more important determinates of memory function than the HF itself. The entorhinal and perirhinal cortices, along with portions of the parahippocampal cortex, also play an important role in memory processing, and these regions may undergo comparable degrees of involutional change with age. Therefore, our finding of a significant correlation between HF size and memory performance does not necessarily imply a causal role for the $\mathrm{HF}$ and may actually reflect degeneration of other medial temporal lobe structures with which the HF shares variance. Although we were unable to measure these other structures in this study, the relative contribution of medial temporal lobe subregions to memory performance in normal aging should be the focus of future research using higher resolution MRI techniques.

Finally, the goal of this study was not to examine the relationship between HF size and models of hippocampal-specific memory processing but, rather, to test whether HF atrophy was related to the general phenomenon of age-associated memory loss. Because delayed secondary memory impairment is a principal and clinically well-documented feature of this phenomenon with clear relevance to hippocampal function, we sought to develop a measure that would effectively represent it as a cognitive construct. By combining several paradigms commonly used clinically to assess memory performance in older adults and applying a statistical method of correction for nondelayed recall, the rDR variable represents an intuitively valid estimate of delay-specific memory function. In addition to nondelayed and delayed memory performance, future research should investigate whether implicit/explicit dissociations and other cognitive features specific to hippocampal memory processing are associated with HF atrophy in normal aging.

This study was based on the examination of carefully screened research volunteers, and caution must be exercised when applying its conclusions to the general elderly community. Nevertheless, the present observations have implications for the evaluation of older individuals with symptoms of mild memory dysfunction and suggest that hippocampal morphometry could play a role in the differential diagnosis of such complaints. Of particular relevance to these findings may be the recently defined clinical syndrome of Age Associated Memory Impairment (AAMI) (Crook et al. 1986). Forty-four percent of our subjects met criteria for this diagnosis on the basis of their subjective impression of a decline in memory performance over a 5- to 10-year interval [Global Deterioration Scale (GDS) $=2$ ] and a score of $>1$ s.D. below the mean for a young adult reference group on the delayed paired associate and delayed paragraph recall subtests of the Guild Memory Test (Gilbert et al. 1968). Interestingly, even when these AAMI subjects were excluded from the analysis, a significant partial correlation was still obtained between $\mathrm{rHF}$ and $\mathrm{rDR}(r=0.43, P<0.05)$.

Our findings suggest that the well-recognized loss of parenchymal brain volume that accompanies human aging may result in quantifiable behavioral manifestations if structures mediating specific neuropsychologic functions are affected severely enough. Further research must examine the histopathologic basis for hippocampal atrophy in normal aging and determine whether this phenomenon constitutes a significant risk for accelerated intellectual decline.

\section{Acknowledgments}

This work was funded in part by grants IP30AG08051, IR29MH44697, IP30MH4386, IR01MH43965, and IR01AG03051 from the National Institutes of Health. We are indebted to Martha Helmers and Anthony Jalandoni for their expert assistance in preparing the figures.

The publication costs of this article were defrayed in part by payment of page charges. This article must therefore be hereby marked "advertisement" in accordance with 18 USC section 1734 solely to indicate this fact.

\section{References}

Brody, H. 1955. Organization of the cerebral cortex III. A study of aging in the human cerebral cortex. /. Comp.

Neurol. 102: 511-556.

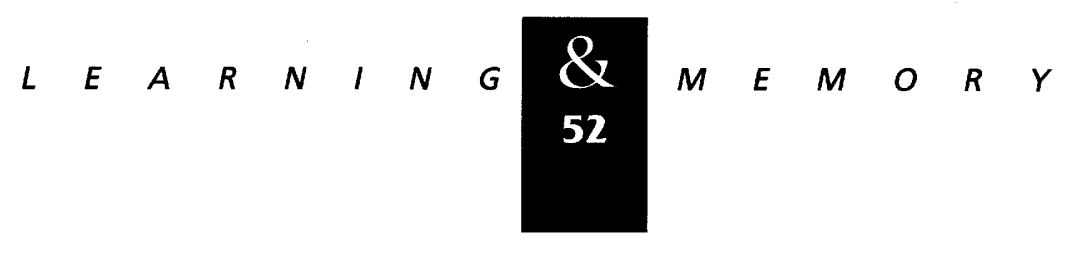


Cockrell, J.R. and M.F. Folstein. 1988. Mini-mental state examination (MMSE). Psychopharmacol. Bull. 24: 689-692.

Cohen, J. and P. Cohen. 1983. Applied multiple regression/correlation analysis for the behavioral sciences. Lawrence Erlbaum Associates, Hillsdale, NJ.

Convit, A., M.J. de Leon, J. Golomb, A.E. George, C.Y. Tarshish, M. Bobinski, W. Tsui, S. de Santi, J. Wegiel, and H. Wisniewski. 1993. Hippocampal atrophy in early Alzheimer's disease, anatomic specificity and validation. Psychiatr. Q. 64: 371-387.

Crook, T., R.T. Bartus, S.H. Ferris, P. Whitehouse, G.D. Cohen, and S. Gershon. 1986. Age-associated memory impairment: Proposed diagnostic criteria and measures of clinical change-Report of a NIMH Work Group. Dev. Neuropsychol. 2: 261-276.

de Leon, M.J., A.E. George, L.A. Stylopoulos, G. Smith, and D.C. Miller. 1989. Early marker for Alzheimer's disease: The atrophic hippocampus. Lancet 2: 672-673.

de Leon, M.J., J. Golomb, A.E. George, A. Convit, C.Y. Tarshish, T. McRae, S. de Santi, G. Smith, and S.H. Ferris. 1993. The radiologic prediction of Alzheimer's disease: The atrophic hippocampal formation. Am. J. Neuroradiol. 14: 897-906.

Ferris, S.H., T. Crook, E. Clark, M. McCarthy, and D. Rae. 1980. Facial recognition memory deficits in normal aging and senile dementia. J. Gerontol. 35: 707-714.

Ferris, S.H., T. Crook, C. Flicker, B. Reisberg, and R.T. Bartus. 1986. Assessing cognitive impairment and evaluating treatment effects: Psychometric performance tests. In The handbook for clinical memory assessment of older adults (ed. L.W. Poon), pp. 139-148. American Psychological Association, Washington, D.C.

Ferris, S.H., C. Flicker, and B. Reisberg. 1988. NYU computerized test battery for assessing cognition in aging and dementia. Psychopharmacol. Bull. 24: 699-702.

Flicker, C., S.H. Ferris, and B. Reisberg. 1993. A longitudinal study of cognitive function in elderly persons with subjective memory complaints. J. Am. Geriatr. Soc. 41: 1071-1074.

Folstein, M.F., S.E. Folstein, and P.R. McHugh. 1975. Mini-mental state: A practical method for grading the cognitive state of patients for the clinician. J. Psychiatr. Res. 12: 189-198.

Fozard, J.L. 1985. Psychology of aging-Normal and pathological age differences in memory. In Textbook of geriatric medicine and gerontology (ed. J.C. Brocklehurst), pp. 122-144. Churchill/Livingstone, Edinburgh, Scotland.

French, J.W., R.B. Ekstrom, and A. Leighton. 1974. First and last names test. In Kit of reference tests for cognitive factors (rev. ed.). Educational Testing Service, Princeton, NJ.
Gilbert, J.G., R.F. Levee, and F.L. Catalano. 1968. A preliminary report on a new memory scale. Percept. Mot. Skills 27: 277-278.

Golomb, J., M.J. de Leon, A. Kluger, A.E. George, C. Tarshish, and S.H. Ferris. 1993. Hippocampal atrophy in normal aging: An association with recent memory impairment. Arch. Neurol. 50: 967-976.

Hachinski, V.C., P. Potter, and H. Mersky. 1987. Leuko-araiosis. Arch. Neurol. 44: 21-23.

Hamilton, M. 1967. Development of a rating scale for primary depression illness Bri. J. Soc. Clin. Psychol. 6: $278-296$.

Jack, C., M. Bently, C. Twomey, and A. Zinsmeister. 1990. $M R$ imaging-based volume measurements of the hippocampal formation and anterior temporal lobe: Validation studies. Radiology 176: 205-209.

Jack, C.R. Jr., R.C. Petersen, P.C. O'Brien, and E.G. Tangalos. 1992. MR-based hippocampal volumetry in the diagnosis of Alzheimer's disease. Neurology 42: 183-188.

Kemper, T. 1984. Neuroanatomical and neuropathological changes in normal aging and in dementia. In Clinical neurology of aging (ed. M.L. Albert), pp. 9-52. Oxford University Press, New York.

Killiany, R.J., M.B. Moss, M.S. Albert, T. Sandor, J. Tieman, and $F$. Jolesz. 1993. Temporal lobe regions on magnetic resonance imaging identify patients with early Alzheimer's disease. Arch. Neurol. 50: 949-954.

Kolb, B. and I.Q. Whishaw. 1990. Head trauma and degenerative diseases. In Fundamentals of human neuropsychology, pp. 830-832. W.H. Freeman, New York.

Matarazzo, J.D. 1972. Wechsler's measurement and appraisal of adult intelligence, 5th ed. Williams and Wilkins, Baltimore, MD.

Moscovitch, M. and G. Winocur. 1992. The neuropsychology of memory and aging. In The handbook of aging and cognition (ed. F.I.M. Craik and T.A. Salthouse), pp. 315-372. Lawrence Erlbaum Associates, Hillsdale, NJ.

Petersen, R.C., G. Smith, E. Kokmen, R.J. Ivnik, and E.G. Tangalos. 1992. Memory function in normal aging. Neurology 42: 396-401.

Pfefferbaum, A., E.V. Sullivan, T.L. Jernigan, R.B. Zipursky, M.J. Rosenbloom, J.A. Yesavage, and J.R. Tinklenberg. 1990. A quantitative analysis of $\mathrm{CT}$ and cognitive measures in normal aging and Alzheimer's disease. Psychiatr. Res. Neuroimaging 35: 115-136.

Poon, L.W. 1985. Differences in human memory with aging: Nature, causes, and clinical implications. In Handbook of the psychology of aging (ed. J.E. Birren and K.W. Schaie), pp. 427-462. Van Nostrand Reinhold, New York.

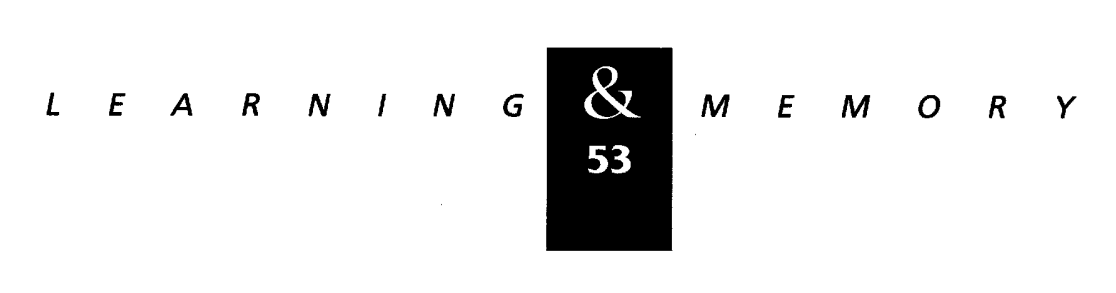


Downloaded from learnmem.cshlp.org on April 26, 2023 - Published by Cold Spring Harbor Laboratory Press Golomb et al.

Press, G.A., D.G. Amaral, and L.R. Squire. 1989.

Hippocampal abnormalities in amnesic patients revealed by high-resolution magnetic resonance imaging. Nature 341: 54-57.

Reisberg, B., S.H. Ferris, M.J. de Leon, and T. Crook. 1982.

The global deterioration scale for assessment of primary degenerative dementia. Am. J. Psychiatr. 139: 1136-1139.

Rosen, W.G., R.D. Terry, P.A. Fuld, R. Katzman, and A. Peck. 1980. Pathological verification of ischemia score in differentiation of dementias. Ann. Neurol. 7: 486-488.

Squire, L.R. and S. Zola-Morgan. 1991. The medial temporal lobe memory system. Science 20: 1380-1386.

Sullivan, E.V., P.K. Shear, D.H. Mathalon, L.O. Kelvin, J.A. Yesavage, J.R. Tinklenberg, and A. Pfefferbaum. 1993.

Greater abnormalities of brain cerebrospinal fluid volumes in younger than in older patients with Alzheimer's disease. Arch. Neurol. 50: 359-373.

Terry, R.D., R. DeTeresa, and L.A. Hansen. 1987.

Neocortical cell counts in normal human adult aging. Ann. Neurol. 21: 530-539.

Wechsler, D.A. 1958. The measurement and appraisal of adult intelligence. Williams and Wilkins, Baltimore, MD.

Received December 10, 1993; accepted in revised form March 4, 1994. 


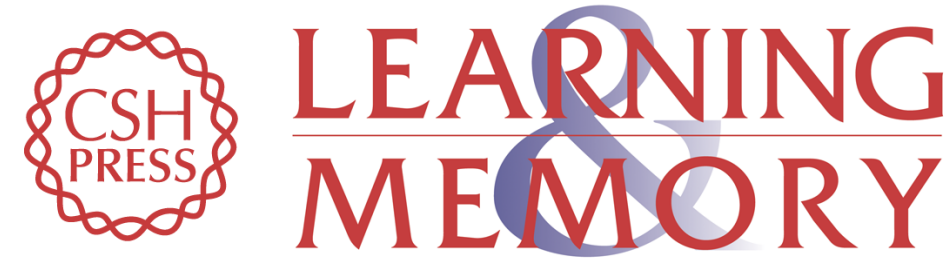

\section{Hippocampal formation size in normal human aging: a correlate of delayed secondary memory performance.}

J Golomb, A Kluger, M J de Leon, et al.

Learn. Mem. 1994, 1:

Access the most recent version at doi:10.1101/lm.1.1.45

References

This article cites 25 articles, 3 of which can be accessed free at: http://learnmem.cshlp.org/content/1/1/45.full.html\#ref-list-1

License

Email Alerting Service

Receive free email alerts when new articles cite this article - sign up in the box at the top right corner of the article or click here. 